\title{
T cell acute lymphoblastic lymphoma complicated with myeloid sarcoma in an adult: A case report
}

\author{
YING WANG, SHUPENG WEN, ZHIYUN NIU, LINA XING, FUXU WANG and XUEJUN ZHANG
}

\author{
Department of Hematology, The Second Hospital of Hebei Medical University, Shijiazhuang, Hebei 050000, P.R. China
}

Received June 10, 2016; Accepted April 13, 2017

DOI: $10.3892 / 01.2017 .7187$

\begin{abstract}
The present case report describes a rare case of T cell acute lymphoblastic lymphoma (T-LBL) in the lymph node with myeloid sarcoma in the pericardium. A 33-year-old Chinese male was admitted to hospital on 4 July 2015 exhibiting a fever and having experienced wheezing and fatigue for the previous 7 days. Routine pathological, computed tomographic, cytological and immunophenotypic observations revealed a diagnosis of T-LBL in the lymph node on 7 August 2015, without evidence of bone marrow (BM) involvement. The patient received induction chemotherapy for T-LBL and achieved partial remission. The patient was identified to have multiple serous effusion and analysis of pericardial effusion cells revealed the diagnosis of T-LBL with extramedullary myeloid sarcoma (without BM involvement) on 25 November 2015. On 30 December 2015, the patient was identified to exhibit proliferation of primary myeloid cells in the peripheral blood and BM, and an abnormal karyotype in BM cells, indicating that the complicated myeloid sarcoma involved the BM. No matched donor was available so the patient received chemotherapy to manage the disease. The patient was discharged on 31 January 2016 and ceased treatment. The patient succumbed on 19 February 2016 at home. To the best of our knowledge, T-LBL complicated with myeloid sarcoma had not been previously reported in Chinese adult male patients. In addition, the involvement of the BM and aberrant karyotype of the complicated myeloid sarcoma in the patient were rare.
\end{abstract}

\section{Introduction}

T-cell acute lymphoblastic leukemia (T-ALL)/T-cell lymphoblastic lymphoma (T-LBL) is a rare and aggressive neoplasm

Correspondence to: Dr Ying Wang or Dr Xuejun Zhang, Department of Hematology, The Second Hospital of Hebei Medical University, 215 Heping West Road, Shijiazhuang, Hebei 050000, P.R. China

E-mail: ywang_2hhmu@163.com

E-mail: zhxjhbmu@126.com

Key words: T cell acute lymphoblastic lymphoma, myeloid sarcoma, bone marrow, serous effusion which originates from T-cell progenitors and may involve the bone marrow (BM) and blood (for T-ALL) or the thymus, nodal and extranodal sites (for T-TBL). T-ALL and T-LBL are similar in morphology and are thus classified together as T-ALL/LBL by the World Health Organization (WHO) (1). With intensified chemotherapy, the prognosis of T-ALL/LBL has markedly improved (2,3). Lymphoblasts in T-ALL/LBL typically express terminal deoxynucleotidyltransferase (TDT) and variably express T-lineage makers, including cluster of differentiation (CD)la, CD2, CD3, CD4, CD5, CD7 and CD8. Immunophenotyping is required to diagnose T-ALL/LBL.

Myeloid sarcoma is a rare extramedullary myeloid neoplasm characterized as a tumor mass consisting of mature or immature myeloid blasts and occurring at almost any anatomical site other than the BM, including the skin, lymph nodes, gastrointestinal tract, bone, soft tissue and testis (4). Myeloid sarcoma with clinical characteristics is diagnosed by morphological observation, immunophenotypical analysis, fluorescent in situ hybridization (FISH) on tissue sections and/or conventional karyotyping of BM or peripheral blood (PB) cells (4). In myeloid sarcoma, CD68 is the most common type of expressed marker, followed by myeloperoxidase (MPO), CD117, CD99, CD68/phosphoglucomutase-1, CD34 and TDT (4). In addition, chromosomal aberrations were determined in $\sim 50 \%$ of myeloid sarcoma cases, including monosomy 7, trisomy 8 and mixed lineage leukemia-splitting; however, $\mathrm{t}(8 ; 21)$ was rarely observed (4). These chromosomal aberrations allow for the distinction of myeloid sarcoma from lymphomas, including LBL, and blastic plasmacytoid dendritic cell neoplasms (5).

Leukemia/lymphoma presentation with myeloid sarcoma is rare. In the present case report, a case of T-LBL with myeloid sarcoma (with serous effusion as the main manifestation) in a Chinese adult male patient is described.

\section{Case report}

A 33-year-old Chinese male was admitted to the Department of Hematology, The Second Hospital of Hebei Medical University (Shijiazhuang, China) on 4 July 2015, presenting with a fever and having experienced wheezing and fatigue for the previous 7 days. The patient was a teacher and was otherwise healthy prior to being admitted. Upon admission, superficial lymph node enlargement in the right neck and bilateral axillar supraclavicular fossa was identified. The patient's heart and lungs 
were normal, without enlargement. Hematological analysis, using an automated hematology analyzer (Sysmex, Kobe, Japan), identified that the patient had a white blood cell count of $8.87 \times 10^{9}$ cells $/ 1$, a hemoglobin level of $13.5 \mathrm{~g} / \mathrm{dl}$ and a platelet count of $438 \times 10^{9}$ cells $/ 1$. Serum levels of lactate dehydrogenase and uric acid were at increased levels of $297 \mathrm{U} / \mathrm{l}$ and $264.8 \mathrm{mg} / \mathrm{dl}$, respectively. Additional laboratory findings were as follows: C-reactive protein, $11 \mathrm{mg} / \mathrm{dl}$; aspartate transaminase activity, $33 \mathrm{U} / \mathrm{l}$; and fibrinogen, $0.82 \mathrm{~g} / \mathrm{l}$. A computed tomography (CT) scan, using a 16-slice spiral CT (GE Healthcare, Chicago, IL, USA), revealed an enhanced density of multiple nodules in the retroperitoneal lymphonodus and in the mediastinum and abdomen (Fig. 1), indicating multiple lymph node enlargement which is characteristic of lymphoma. Immunohistochemical staining for paired box (PAX)-5, MPO, CD20, CD21, CD3, TDT, CD7, CD30 and Ki67 was performed on the lymph node, using the following monoclonal antibodies: Rabbit anti-PAX-5 (cat. no. TA322317, 1:50), rabbit anti-MPO (cat. no. TA349402, 1:100), rabbit anti-CD20 (cat. no. TA353671, 1:50), rabbit anti-CD21 (cat. no. TA327628, 1:100), rabbit anti-CD3 (cat. no. AP22689PU-N, 1:50), rabbit anti-TDT (cat. no. TA327743, 1:100), rabbit anti-CD7 (cat. no. TA353715, 1:100), rabbit anti-CD30 (cat. no. TA590389, 1:150) and mouse anti-Ki67 (cat. no. TA803157, 1:150). All antibodies were purchased from OriGene Technologies (Beijing, China). Immunohistochemical staining was performed as follows: The slide was rinsed twice for $5 \mathrm{~min}$ in TBS $0.025 \%$ Triton with gentle agitation, then incubated in $0.3 \% \mathrm{H}_{2} \mathrm{O}_{2}$ in TBS for $15 \mathrm{~min}$ at room temperature. Subsequently, the slide was blocked in $10 \%$ normal serum with $1 \%$ bovine serum albumin (BSA) in TBS for $2 \mathrm{~h}$ at room temperature. The primary antibody was then applied diluted in TBS with $1 \%$ BSA to the slide and incubated overnight at $4^{\circ} \mathrm{C}$. Subsequently, Rabbit Anti-Mouse IgG $\mathrm{H} \& \mathrm{~L}$ horseradish peroxidase conjugated (HRP; 1:1,000; ab6728; Abcam, Cambridge, UK) or Goat anti-Rabbit IgG H\&L HRP (1:1,000; ab6721; Abcam) antibodies were added to the slide, diluted in TBS with $1 \%$ BSA and incubated for $1 \mathrm{~h}$ at room temperature. The slide was then rinsed in running tap water for $5 \mathrm{~min}, 2$ times. The DAB color test was performed using a DA1010 DAB kit (x20), according to the protocol of the DA1010 DAB Color test kit (Beijing solaibotechnology Co. Ltd., Beijing, China). Finally, following DAB coloring, the slide was sealed and images captured using a LEICA DM 2000 light microscope at magnifications, x100 and $\mathrm{x} 400$. Staining was identified to be negative for the B-cell markers PAX-5, MPO and CD20, but positive for the T-cell markers TDT, CD7, CD30 (sporadic +), CD3 (partial +), Ki67 $(50 \%+)$ and CD21 in the lymph node (Fig. 2A). These results were consistent with the immunophenotypical characteristics of T-LBL. Hematoxylin and eosin staining revealed pathological changes including viability, marked nuclear atypia and frequent karyokinesis of cells in the lymph node (Fig. 2B).

A PB smear identified that neutrophilic metamyelocytes were occasionally observed $(\sim 2.6 \%)$ in PB cells and a BM smear revealed a limited proportion of myeloblasts $(1 \%)$ and promyelocytes (1\%) among the nucleated BM cells. Karyotype analysis, using the $\mathrm{R}$ banding technique (6), indicated a normal karyotype (46 XY) of the BM cells (Fig. 2C). These results revealed that the $\mathrm{PB}$ and $\mathrm{BM}$ were not involved. Flow cytometric analysis, using a BD FACSCanto II $^{\mathrm{TM}}$ instrument (BD
Biosciences, Franklin Lakes, NJ, USA) revealed that, among the nucleated BM cells, the majority were positive for CD20 (93.3\%) and CD22 (97.3\%), and between 33 and 50\% were positive for CD38 (35.3\%), CD79b (38.5\%), CD20 (49.3\%), CD24 (42.8\%), $\kappa(36.9 \%)$ and $\lambda(30.9 \%)$. In contrast, a limited number of nucleated BM cells were positive for CD19 (1.5\%) and $\mathrm{CD}^{+} \mathrm{CD} 23^{+}$cells were identified $(2.5 \%$; characteristic of B-cell lymphoma/leukemia), which enabled a diagnosis of B-cell lymphoma to be excluded. CD103, CD11c and CD25 were not determined in BM cells, thus excluding the diagnosis of hairy cell leukemia, a type of B-cell lymphoma. In addition, granulocyte series accounted for $33 \%$ of the nucleated $\mathrm{BM}$ cells, thus excluding the presence of abnormal early myeloid cells. The patient was diagnosed with mediastinal T-LBL, without evidence of the involvement of the BM on 7 August 2015.

The patient received induction chemotherapy with dexamethasone (15 mg/day on days $1-5)$, vincristine $\left(1.4 \mathrm{mg} / \mathrm{m}^{2} /\right.$ day on day 1$)$, daunorubicin $\left(50 \mathrm{mg} / \mathrm{m}^{2} /\right.$ day on day 1$)$, cyclophosphamide $\left(750 \mathrm{mg} / \mathrm{m}^{2} /\right.$ day on day 1$)$ and L-asparaginase $\left(6,000 \mathrm{U} / \mathrm{m}^{2} /\right.$ day on days $1,3,5,7,9$ and 11$)$ and achieved partial remission subsequently. After 2 weeks, the patient received a course of dexamethasone (15 mg/day on day 1-5), vincristine $\left(1.4 \mathrm{mg} / \mathrm{m}^{2} /\right.$ day on day 1$)$, daunorubicin $\left(50 \mathrm{mg} / \mathrm{m}^{2} /\right.$ day on day 1) and cyclophosphamide $\left(750 \mathrm{mg} / \mathrm{m}^{2} /\right.$ day on day 1$)$. On 17 November 2015, multiple serous effusion (including pericardial effusion, pleural effusion and ascites) was identified. Immunophenotypical analysis, using flow cytometry, revealed that the blast cells accounted for $25 \%$ of the nucleated BM cells, which were positive for T-LBL cell markers (including CD33, CD34, CD38, HLA-DR, CD123, CD56, CD11c and CD15) and negative for B-LBL cell markers (including MPO, CD117, CD16, CD2, CD5, cytosolic CD3, CD7, CD64, CD14, CD19, CD10, CD20, CD22 and CD79a). These results confirmed the diagnosis of T-LBL in the patient. The polymerase chain reaction (PCR) demonstrated that acute myeloid leukemia 1-eight-twenty-one (AML1-ETO) fusion protein was expressed in pericardial effusion cells (Fig. 3A), characteristic of the abnormal clone of myeloid sarcoma (as a type of myeloid leukemia). The following genes were not determined using FISH (DNA Technology, Aarhus, Denmark): Breakpoint cluster region-Abelson murine leukemia viral oncogene homolog 1; promyelocytic leukemia protein-retinoic acid receptor $\alpha$; myosin heavy chain 11-core-binding factor $\beta$ subunit; mixed-lineage leukemia 1; platelet-derived growth factor receptor $\alpha$ (PDGFRA); platelet-derived growth factor receptor $\beta$ (PDGFRB); and fibroblast growth factor $\alpha 1$ (FGFA1). In addition, aberrant karyotype $t(8 ; 21)$ was identified in pericardial effusion cells (Fig. 3B). Therefore, the patient was diagnosed with T-LBL complicated with extramedullary myeloid sarcoma (in the pericardium) on 25 November 2015, according to the 2008 WHO classification of Tumors of Haematopoietic and Lymphoid Tissues (7).

The patient received a second course of induction chemotherapy with cytarabine $\left(1.0 \mathrm{~g} / \mathrm{m}^{2} /\right.$ day, every $12 \mathrm{~h}$ for 3 days $)$ and mitoxantrone ( $8 \mathrm{mg} / \mathrm{m}^{2} /$ day for 3 days), and was expected to receive allogeneic hematopoietic stem cell transplantation. However, since there were no matched donors available and the patient was experiencing pericardial effusion, following chemotherapy with mitoxantrone and cytarabine, the patient 

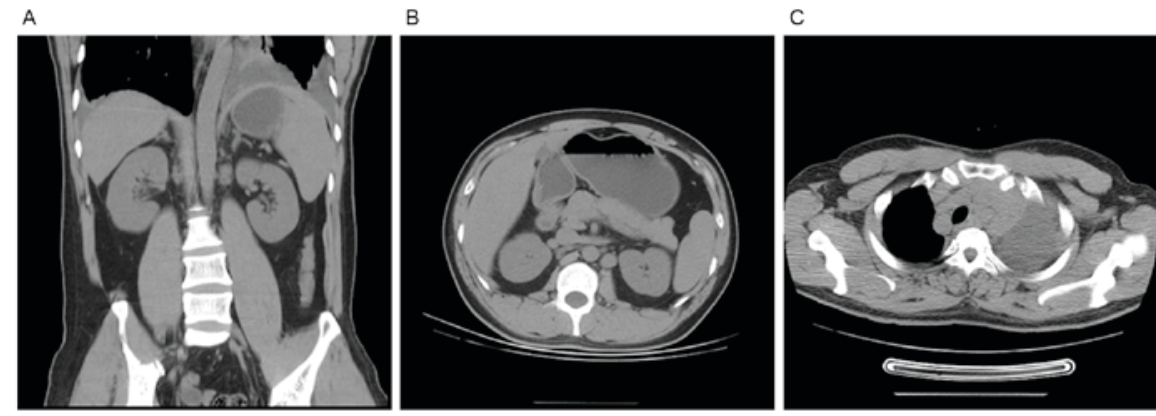

Figure 1. Computed tomographic scan demonstrating multiple lymph node enlargement. (A) Retroperitoneal lymph node enlargement. (B) Abdominal lymph node enlargement. (C) Mediastinal lymph node enlargement.

A

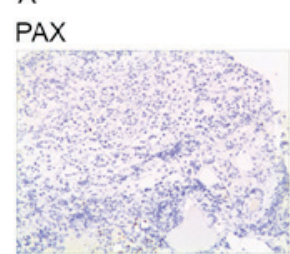

TDT

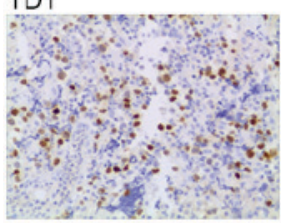

CD3

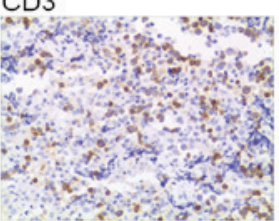

MPO



CD7
$\mathrm{CD} 20$



$\mathrm{CD} 30$

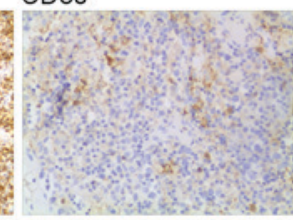

$\mathrm{CD} 21$

Ki67
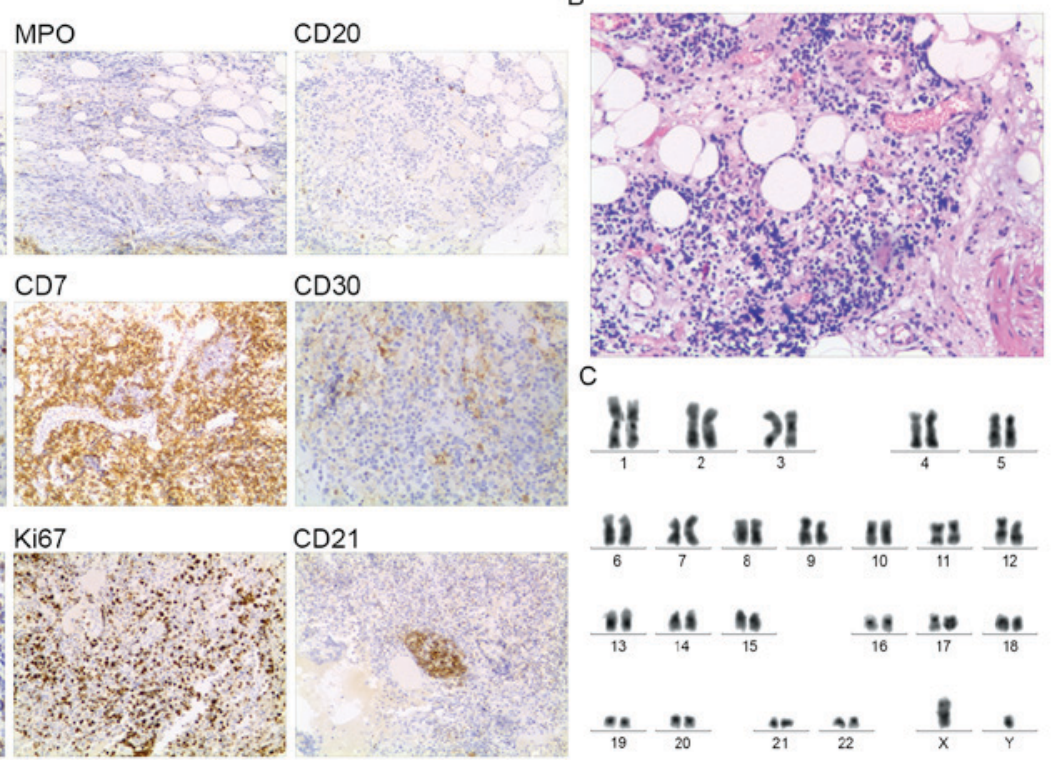

Figure 2. Immunohistological, histological, karyotypic and flow cytometric analysis indicated the diagnosis of T-LBL in a patient, without the involvement of the bone marrow (7 August 2015). (A) Immunohistological observation of lymph node (magnification, x200). (B) Histological analysis of lymph node (magnification, x200). (C) Karyotype analysis of the bone marrow cells, using the R banding technique revealed the normal karyotype (46,XY). PAX, paired box; MPO, myeloperoxidase; $\mathrm{CD}$, cluster of differentiation; TDT, terminal deoxynucleotidytransferase.

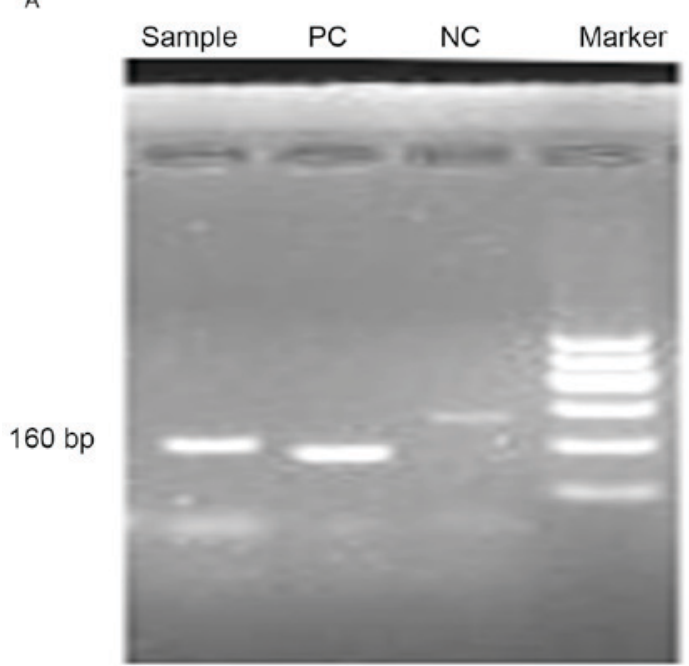

B
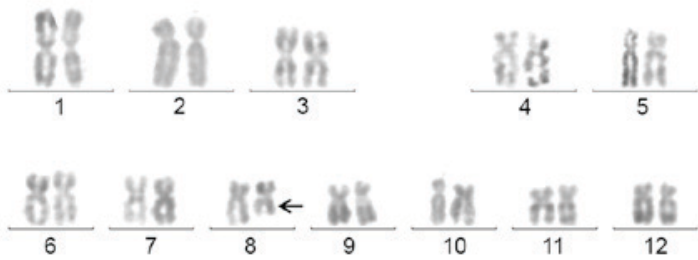

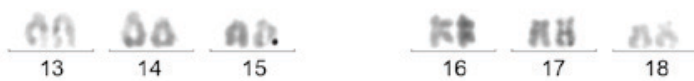

$\frac{\mathbb{B} \boldsymbol{K}}{19} \frac{\mathrm{3} \boldsymbol{B}}{20} \quad \frac{4 \mathrm{~A} \leftarrow}{21} \frac{\mathrm{BA}}{22}$

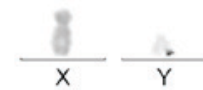

Figure 3. PCR detection and karyotype analysis of pericardial effusion cells (25 November 2015). (A) PCR detection of AML1-ETO in pericardial effusion cells, DNA sample from pericardial effusion cells. AML1-ETO-containing plasmid (positive control) and purified water (negative control) underwent PCR, and the products underwent agarose electrophoresis. (B) Abnormal karyotype $\mathrm{t}(8 ; 21)$ (arrows) in pericardial effusion cells. PCR, polymerase chain reaction; AML1-ETO, acute myeloid leukemia 1-eight-twenty-one fusion; PC, positive control; NC, negative control. 


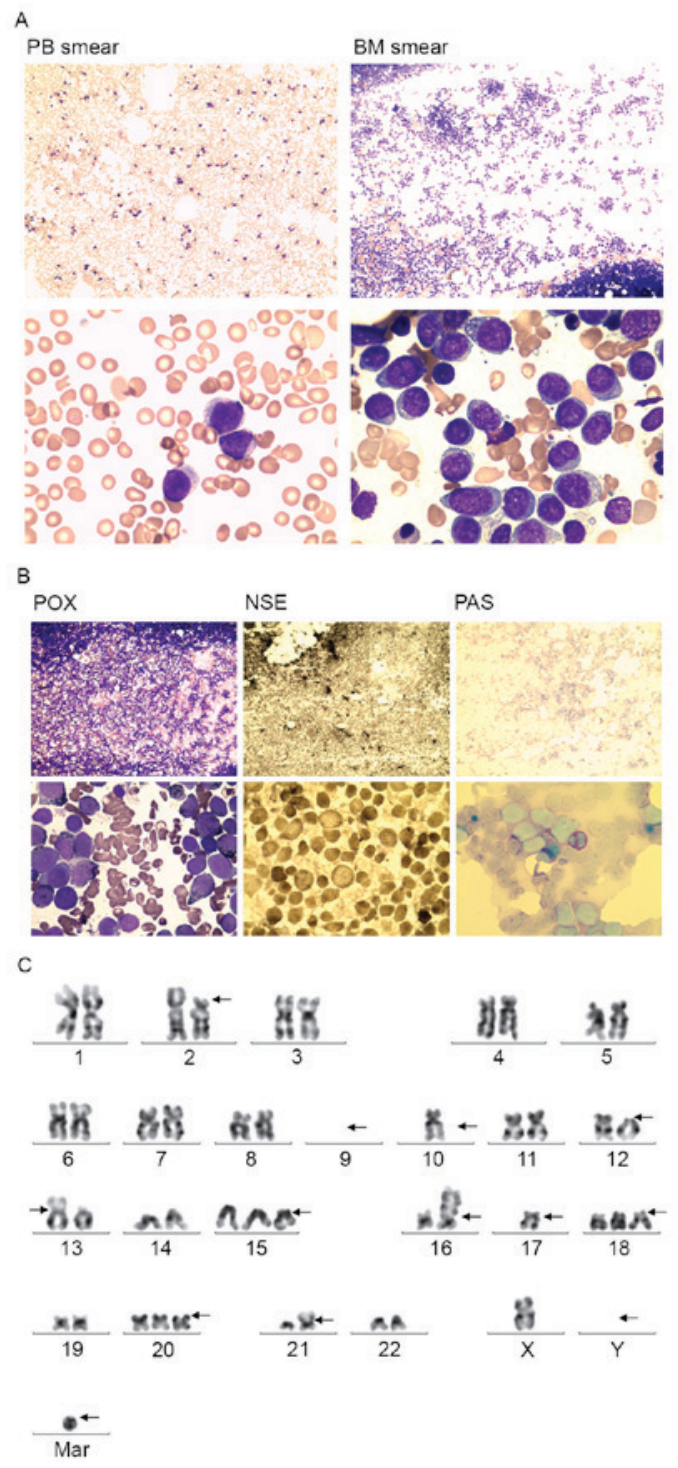

Figure 4. BM and PB smear and karyotype analysis. (A) BM smear identified primary myeloid cells proliferated which were positive for POX and NSE, and negative for PAS. (B) Wright's Giemsa staining. (C) Abnormal karyotype of the bone marrow cells (28 December 2015); arrows show complex chromosomal rearrangements. BM, bone marrow; PB, peripheral blood; POX, peroxidase; NSE, non-specific esterase; PAS, periodic acid-Schiff.

was advised to receive local radiotherapy. On 28 December 2015, following three doses of local radiotherapy (50 cGy/dose), the patient was required to stop radiation as the white blood cells increased to $40.0 \times 10^{9}$ cells/1. BM and PB samples were collected, and the BM and PB smears identified the proliferation of primary myeloid cells (Fig. 4A) which were positive for peroxidase, non-specific esterase and periodic acid-Schiff staining (Fig. 4B). Chrom osomal analysis identified an abnormal karyotype of the BM cells as follows: 42,X,-Y,del (2)(p12),-9,-9, del(12)(p11),der(13;17)(q10;q10), add(16)(p13), add (21)(p11)[3]/43,idem,+mar[8]/45,idem,-10,+15,+18,+20,+mar[4] (Fig. 4C). On 30 December 2015, the patient was diagnosed with T-LBL complicated with myeloid sarcoma, which involved the BM. Furthermore, Second Generation High-Throughput Sequencing was then carried out using a MiSeq platform (Illumina Inc., San Diego, CA, USA) and the sequences generated were run on Human Genome Version 19 using Illumina bcl2fastq version 2.15 software (Illumina, Inc.). Three mutations were identified in the pericardial effusion cells and the BM cells as follows: Tet methylcytosine dioxygenase (TET) 2 NM_001127208:exon11:c.4789_4793del:p.F1597fs mutation; AML1 NM_001754:exon5:c.496_497insGGTTCGGAG TTGCGTGTCG:p.R166fs mutation; and tumor suppressor protein (TP) 53 NM_000546:exon6:c.T581A:p.L194H mutation. The patient subsequently received chemotherapy to control the disease. According to the acute myeloid leukemia (myeloid sarcoma) therapeutic protocol for T-LBL with multiple serous effusion (8), the patient was administered with mitoxantrone ( $8 \mathrm{mg} / \mathrm{m}^{2} /$ day for 3 days) and cytosine arabinoside (100 $\mathrm{mg} / \mathrm{m}^{2} /$ day for 7 days), in combination with L-asparaginase $\left(6,000 \mathrm{U} / \mathrm{m}^{2} /\right.$ day on days $1,3,5,7,9$ and 11). In addition, since mutations of the TET2, AML1 and TP53 genes were identified, the patient was administered decitabine (20 mg/m²/day, via an intravenous drip on days 1-5), a DNA methylation inhibitor used to treat MDS/AML with TP53 and TET2 gene mutations (9). Subsequently, the multiple serous effusion was markedly decreased; however, myeloid blast cells (33\% of the total cells) were observed in a BM smear, indicating that the patient had improved but did not achieve remission. The patient was discharged on 31 January 2016 and ceased treatment. The patient succumbed on 19 February 2016, at home.

The present case report was approved by the Ethics Committee of the Second Hospital of Hebei Medical University (Shijiazhuang, China) and informed consent was obtained from the patient.

\section{Discussion}

The patient was diagnosed with T-LBL using CT scans and pathological, cytological and phenotypical observations on 7 August 2015. However, the corresponding treatment regimen only achieved partial remission, which may be because the treatment was aimed at T-LBL only, despite the patient additionally exhibiting myeloid sarcoma. Subsequently, on 25 November 2015, PCR, FISH and karyotype analysis of the pericardial effusion cells were carried out which identified myeloid sarcoma. Since PDGFRA, PDGFRB and FGFA1, associated with myeloproliferative and lymphoid neoplasms (10-12), were all negative in the pericardial effusion cells, a diagnosis of myeloproliferative and lymphoid neoplasms was excluded. Since the interval between the diagnosis of T-LBL and that of myeloid sarcoma was <1 year (between August and November 2015), the patient did not meet the characteristics of T-acute myelocytic leukemia $(13,14)$ and was subsequently diagnosed with T-LBL complicated with myeloid sarcoma, which is markedly rare.

A number of patients with T-LBL may subsequently develop myeloid sarcoma (15); however, T-ALL/LBL with myeloid sarcoma is rare and has only been described in a limited number of cases $(5,16)$. A previous study identified a 14-year-old male who presented with chronic myelogenous leukemia (CML) in the blast phase with segregated extramedullary (nodal) myeloid sarcoma and T-LBL (5). In addition, a 49-year-old female patient with CML was identified to exhibit myeloid sarcoma and T-LBL (16). In the present case report, a 33-year-old Chinese male patient was described who presented with primary T-LBL. The patient was diagnosed with myeloid 
sarcoma during the treatment course ( 3 months following the diagnosis of T-LBL) and, $\sim 1$ month subsequently, myeloid sarcoma which involved the BM. It is not possible for myeloid sarcoma to develop from T-LBL in such a short period; therefore, it is hypothesized that myeloid sarcoma, in this patient, did not develop from T-LBL, but was complicated with T-LBL during the pathogenesis. To the best of our knowledge, this is the first case of T-LBL with myeloid sarcoma in a Chinese adult male patient.

Typically, myeloid sarcoma occurs at almost any anatomical site, with the exception of the BM (4). In the present case report, the patient with T-LBL and myeloid sarcoma was identified to exhibit involvement of the BM. The diagnosis of T-LBL excludes the possibility of the involvement of the $\mathrm{BM}$ in T-LBL; therefore, the involvement of the BM, observed 1 month after the primary detection of myeloid sarcoma, may be attributable to myeloid sarcoma. Involvement of the BM in the complicated myeloid sarcoma and the aberrant karyotype $\mathrm{t}(8 ; 21)$ in complicated myeloid sarcoma is rarely observed.

Notably, three mutations were identified (TET2, AML1 and TP53 genes) in the pericardial effusion cells and BM cells (TET2 NM_001127208:exon11:c.4789_4793del:p.F1597fs; AML1 NM_001754:exon5:c.496_497insGGTTCGGAGT TGCGTGTCG:p.R166fs; and TP53 NM_000546:exon6:c. T581A:p.L194H). It is hypothesized that mutations of the TET2, AML1 and TP53 genes may participate in a common pathway attributable to the pathogenesis of T-LBL and myeloid sarcoma. Additional studies are required to explore the underlying molecular mechanisms of the pathogenesis of T-LBL complicated with myeloid sarcoma. The present case report described a rare case of T-LBL complicated with myeloid sarcoma (with distinct BM involvement and karyotype) in a Chinese adult male patient.

\section{Acknowledgements}

The present case report was supported by the Hebei Provincial Natural Science Foundation of China (grant no. H2016206577).

\section{References}

1. Steven H, Elias, Campo NancyLee, Harris, et al: International Agency for Research on Cancer Lyon. WHO Classification of Tumours of Haematopoietic and Lymphoid Tissues, pp176, 2008.

2. Thomas DA, O'Brien S, Cortes J, Giles FJ, Faderl S, Verstovsek S, Ferrajoli A, Koller C, Beran M, Pierce S, et al: Outcome with the hyper-CVAD regimens in lymphoblastic lymphoma. Blood 104 1624-1630, 2004.
3. Marks DI, Paietta EM, Moorman AV, Richards SM, Buck G, DeWald G, Ferrando A, Fielding AK, Goldstone AH, Ketterling RP, et al: T-cell acute lymphoblastic leukemia in adults: Clinical features, immunophenotype, cytogenetics, and outcome from the large randomized prospective trial (UKALL XII/ECOG 2993). Blood 114: 5136-5145, 2009.

4. Pileri SA, Ascani S, Cox MC, Campidelli C, Bacci F, Piccioli M, Piccaluga PP, Agostinelli C, Asioli S, Novero D, et al: Myeloid sarcoma: Clinico-pathologic, phenotypic and cytogenetic analysis of 92 adult patients. Leukemia 21: 340-350, 2007.

5. Chen X, Rutledge JC, Wu D, Fang M, Opheim KE and Xu M: Chronic myelogenous leukemia presenting in blast phase with nodal, bilineal myeloid sarcoma and T-lymphoblastic lymphoma in a child. Pediatr Dev Pathol 16: 91-96, 2013.

6. Verma RS and Lubs HA: A simple R banding technic. Am J Hum Genet 27: 110-117, 1975 .

7. Borowitz MJ and Chan JKC: T lymphoblastic leukaemia/lymphoma. In: Swerdlow SH, Campo E, Harris NL, Jaffe ES, Pileri SA, Stein H, et al (eds): WHO classification of tumours of haematopoietic and lymphoid tissues. 4th edition. IARC Press Lyon, pp176-178, 2008.

8. Takahashi H, Koh K, Kato M, Kishimoto H, Oguma E and Hanada R: Acute myeloid leukemia with mediastinal myeloid sarcoma refractory to acute myeloid leukemia therapy but responsive to L-asparaginase. Int J Hematol 96: 136-140, 2012.

9. Bejar R, Lord A, Stevenson K, Bar-Natan M, Pérez-Ladaga A, Zaneveld J, Wang H, Caughey B, Stojanov P, Getz G, et al: TET2 mutations predict response to hypomethylating agents in myelodysplastic syndrome patients. Blood 124: 2705-2712, 2014.

10. Bain BJ and Fletcher SH: Chronic eosinophilic leukemias and the myeloproliferative variant of the hypereosinophilic syndrome. Immunol Allergy Clin North Am 27: 377-388, 2007.

11. Metzgeroth G, Walz C, Score J, Siebert R, Schnittger S, Haferlach C, Popp H, Haferlach T, Erben P, Mix J, et al: Recurrent finding of the FIP1L1-PDGFRA fusion gene in eosinophilia-associated acute myeloid leukemia and lymphoblastic T-cell lymphoma. Leukemia 21: 1183-1188, 2007.

12. Tokita K, Maki K, Tadokoro J, Nakamura Y, Arai Y, Sasaki K, Eguchi-Ishimae M, Eguchi M and Mitani K: Chronic idiopathic myelofibrosis expressing a novel type of TEL-PDGFRB chimaera responded to imatinib mesylate therapy. Leukemia 21: 190-192, 2007.

13. Pedersen-Bjergaard J, Christiansen DH, Desta F and Andersen MK: Alternative genetic pathways and cooperating genetic abnormalities in the pathogenesis of therapy-related myelodysplasia and acute myeloid leukemia. Leukemia 20: 1943-1949, 2006.

14. Smith SM, Le Beau MM, Huo D, Karrison T, Sobecks RM, Anastasi J, Vardiman JW, Rowley JD and Larson RA: Clinical-cytogenetic associations in 306 patients with therapy-related myelodysplasia and myeloid leukemia: The university of Chicago series. Blood 102: 43-52, 2003.

15. Abruzzo LV, Jaffe ES, Cotelingam JD, Whang-Peng J, Del Duca V Jr and Medeiros LJ: T-cell lymphoblastic lymphoma with eosinophilia associated with subsequent myeloid malignancy. Am J Surg Pathol 16: 236-245, 1992.

16. Krishnan S, Sabai K, Chuah C and Tan SY: Bilineal T lymphoblastic and myeloid blast transformation in chronic myeloid leukemia with TP53 mutation-an uncommon presentation in adults. Curr Oncol 21: e147-e150, 2014. 